\title{
SÍNDROME DE BURNOUT EM PROFISSIONAIS DE SAÚDE EM CENÁRIO DE PANDEMIA DE COVID-19: ANÁLISE DE UM HOSPITAL UNIVERSITÁRIO
}

\section{ARTIGO ORIGINAL}

SALVIATO, Lais Spinelli ${ }^{1}$, VASCONCELOS FILHO, Paulo de Oliveira Vasconcelos ${ }^{2}$

SALVIATO, Lais Spinelli. VASCONCELOS FILHO, Paulo de Oliveira. Síndrome de Burnout em profissionais de saúde em cenário de pandemia de Covid-19: análise de um hospital universitário. Revista Científica Multidisciplinar Núcleo do Conhecimento. Ano 06, Ed. 08, Vol. 06, pp. 27-44. Agosto de 2021. ISSN: 24480959, Link de acesso:

https://www.nucleodoconhecimento.com.br/psicologia/hospital-universitario, $\quad$ DOI: 10.32749/nucleodoconhecimento.com.br/psicologia/hospital-universitario

\section{RESUMO}

Introdução: A Síndrome de Burnout (SB) ocorre quando há o esgotamento físico e emocional do trabalhador. Pesquisas demonstraram que as características do trabalho estão associadas ao desenvolvimento desta doença. A pandemia do coronavírus 2019 (COVID-19) foi responsável por estabelecer novos desafios para os profissionais de saúde relacionados ao surgimento de uma nova doença, como: cargas de trabalho prolongadas e sentimento de insegurança quanto a uma possível infecção pelo vírus. Questão norteadora: Qual a prevalência da SB entre os profissionais de saúde que atuam na linha de frente no combate à COVID-19? Objetivo: O objetivo desta pesquisa foi analisar a repercussão da pandemia de COVID-19 no desenvolvimento da SB entre os trabalhadores da saúde atuando no combate à doença. Metodologia: O levantamento foi realizado integralmente por meio digital. A pesquisa utilizou o Inventário de Burnout de Maslach para evidenciar

\footnotetext{
${ }^{1}$ Graduanda de ensino superior.

2 Pós-doutor.
}

RC: 95817

Disponível em: https://www.nucleodoconhecimento.com.br/psicologia/hospitaluniversitario 
a presença da síndrome entre os participantes, e relacionou as informações obtidas com dados de um questionário sociodemográfico para analisar seu impacto no desenvolvimento da SB. Resultados: Ao todo, 73 pessoas participaram do estudo, composto principalmente de enfermeiros, técnicos de enfermagem e médicos, dos quais $83,5 \%$ eram mulheres. O estudo demonstrou alta prevalência de despersonalização entre os participantes (média de 65,51\%, maior em médicos com $73,3 \%$ ) e moderada prevalência de exaustão emocional (média de 48,27\%; maior em técnicos de enfermagem, com $56,52 \%$ ). Já a baixa realização pessoal foi evidenciada em $32,75 \%$ dos participantes, que contribuiu para que a prevalência da SB entre os profissionais de saúde do hospital estudado ficasse em $14 \%$. Conclusão: Fatores como: o sentimento de realização pessoal pela atuação na pandemia, as estratégias de gestão visando diminuir a sobrecarga dos profissionais e as características próprias do funcionamento da instituição analisada podem justificar a baixa prevalência da síndrome nesse cenário.

Palavras-chave: Síndrome de Burnout, COVID-19, profissionais de saúde.

\section{INTRODUÇÃO}

A doença do coronavírus 2019 (COVID-19) foi relatada pela primeira vez no fim de dezembro de 2019, na China, e logo alcançou escala mundial (LIU et al., 2020). O coronavírus é um betacoronavírus e foi denominado SARS-Cov-2 (LIU et al., 2020). A principal característica da enfermidade é uma pneumonia viral com alta taxa de infecção. Aproximadamente $15 \%$ dos pacientes apresentam o estado grave da doença, com necessidade de internação hospitalar (WHO, 2021). A pandemia forçou a remodelação dos serviços de saúde, com unidades específicas para o tratamento de COVID-19. Pesquisas sobre saúde mental de profissionais da saúde que tratam a COVID-19 indicam que aqueles que estão na linha de frente experimentam uma grande carga psicológica e apresentam resultados referentes à saúde mental muito desfavoráveis, como depressão, ansiedade e insônia (LAl et al., 2020; SASANGOHAR et al., 2020). 
A Síndrome de Burnout (SB) foi identificada no início dos anos 1970 (MASLACH e JACKSON, 1981). Maslach e Jackson, por meio do Inventário de Burnout de Maslach (MBI), realizaram uma investigação sobre as três dimensões que compõem a síndrome: a exaustão emocional, a despersonalização (distanciamento em relação a própria personalidade e atitudes) e o reduzido senso de realização pessoal (MASLACH e JACKSON, 1984; MASLACH et al., 2001). O MBI é a ferramenta padrão para medir a SB em pesquisas. As pessoas que experimentam as três dimensões estão propensas a desenvolver a SB, embora o fator central da síndrome seja a exaustão emocional (MASLACH et al., 2001; TAMAYO et al., 2002). Trabalhos de alto estresse podem levar mais facilmente à SB do que aqueles que têm menos estresse (CODO, 2003). Os profissionais de saúde são frequentemente propensos à SB (EMBRIACO et al., 2007). A síndrome é caracterizada pelo esgotamento físico e emocional do profissional e ocorre quando o indivíduo não possui mais estratégias para enfrentar as situações e conflitos no trabalho (CODO, 2003). Portanto, a SB ocorre quando há uma incapacidade de lidar com o estresse emocional no trabalho ou quando se faz uso excessivo de energia mental e física, o que leva a sentimentos de fracasso e exaustão (MASLACH et al., 2001). Diversas pesquisas apontam que as características do ambiente de trabalho e do trabalhador estão associadas ao desenvolvimento da SB (TRINDADE et al., 2010; EMBRIACO et al., 2007; EBLING et al., 2012). Sintomas clínicos da SB são inespecíficos e incluem cansaço, dores de cabeça, problemas com alimentação, insônia, irritabilidade, instabilidade emocional, e rigidez nas relações com outras pessoas. Grandes variações na prevalência da SB em profissionais da saúde têm sido relatadas tanto em enfermeiros como em médicos (EMBRIACO et al., 2007; GREENGLASS et al., 2001; ROTENSTEIN et al., 2018). O clima no local de trabalho e a carga de trabalho foram determinantes de SB (CODO, 2003).

Uma pesquisa realizada com os profissionais de saúde de cuidados intensivos durante a pandemia mostrou que, em quase 9.500 prestadores, o estresse médio autorreportado, que foi mensurado por um instrumento específico do estudo, apresentou um aumento do escore avaliado de 3 para 8 após a COVID-19 (SCCM, 
2020). Foram relatados como motivos de estresse: a falta de equipamentos de proteção individual, o medo de contrair a doença e o medo de espalhar a infecção para os familiares (SHANAFELT et al., 2020). Além dessas preocupações, também ocorrem conflitos quando há a solicitação para cuidar de pacientes em estado mais grave sem ter o devido conhecimento ou capacitação para tal (CHUNG et al., 2020). Esses estressores relacionados ao trabalho têm sido associados à ansiedade e à depressão e refletem riscos físicos e emocionais reais enfrentados pelos profissionais da linha de frente (EBLING et al., 2012).

Em virtude da velocidade de propagação da pandemia de COVID-19, alguns profissionais da saúde ficaram expostos à contaminação e muitos acabaram por desenvolver a forma grave da doença. Esse fato também leva a uma sensação de insegurança que pode ter aumentado a tensão e o estresse laboral dos profissionais envolvidos diretamente na assistência. O propósito deste estudo foi identificar a prevalência da SB em profissionais da saúde de um hospital universitário que atuam no combate à COVID-19, e como a pandemia impactou na prevalência da síndrome entre os profissionais que atuam na linha de frente.

\section{METODOLOGIA}

O projeto foi revisado e aprovado pela Comissão de Ética em Pesquisa (CEP) da Universidade Federal de São Carlos (UFSCar) sob o número CAAE 4.053.302.

Este é um estudo descritivo com desenho transversal e abordagem quantitativa realizado com os profissionais da saúde que atuam no Hospital Universitário da Universidade Federal de São Carlos, onde, durante a pandemia, foi feita uma adequação para o atendimento de pacientes com COVID-19.

Além do Inventário de Burnout Maslach adequado para cuidados de saúde/cuidadores ou serviços humanos/sociais (MBI-HSS), foi elaborado um questionário para a coleta de dados sociodemográficos. Tanto o questionário quanto o MBI-HSS e o Termo de Consentimento Livre e Esclarecido (TCLE) foram 
transformados em um formulário on-line e disponibilizados no e-mail de um total de 293 profissionais e dez estudantes. Assim, a coleta de dados foi realizada por meio de um formulário autopreenchido que só podia ser acessado com o consentimento do TCLE. A primeira parte coletou dados demográficos. A amostra final contou com 71 profissionais e 2 estudantes. A coleta de dados foi realizada entre junho e setembro de 2020. As chefias e os participantes da instituição foram informados da natureza voluntária, anônima e confidencial do estudo, e foi assegurado que os dados coletados não seriam utilizados para fins de avaliação individual ou institucional.

O MBI-HSS é uma referência universal para avaliar a SB institucional e foi traduzido para o português, adaptado e validado por Lautert (1997), Tamayo (1997) e outros. O MBI avalia as três dimensões da SB. É uma escala tipo Likert de sete pontos com 22 questões: nove questões de exaustão emocional (EE), cinco para despersonalização (DE) e oito para realização pessoal reduzida (RP), esta última com pontuação reversa. $O$ questionário $\mathrm{MBI}$ pode indicar se uma pessoa tende a apresentar SB ou se já tem SB. Para a SB manifestada, as três dimensões devem estar presentes, ou seja, indivíduos que manifestam SB têm altas classificações para EE e DE e baixa classificação para RP. As dimensões devem ser pontuadas separadamente, porque a contribuição de cada dimensão da síndrome é desconhecida. Quando todas as dimensões são negativas, o risco de SB é reduzido. O risco aumenta à medida que os domínios se tornam positivos (MASLACH et al., 2001).

Para análise de dados, foi utilizado o Pacote Estatístico das Ciências Sociais (SPSS), versão 25 (IBM SPSS STATISTICS, 2017). Além das técnicas básicas de análise exploratória como média, desvio padrão e frequência, as diferenças de proporções foram testadas pelo teste de Pearson (qui-quadrado). Para estudar os fatores relacionados aos três aspectos do MBI-HSS (EE, DE e RP), foram feitos cálculos separadamente para cada categoria. As odds ratios (OR) foram calculadas com intervalos respectivos de $95 \%$ de confiança (IC - 95\%). O teste de Wald foi aplicado para obter estimativas de associação de variáveis sociodemográficas com

RC: 95817

Disponível em: https://www.nucleodoconhecimento.com.br/psicologia/hospitaluniversitario 
dimensões da SB. O nível de significância ( $\alpha$ ) foi fixado em $5 \%$, sendo considerados valores significativos de $p<0,05$ e intervalo de confiança.

\section{RESULTADOS}

Um total de 71 questionários foi preenchido por profissionais do hospital e 2 por internos de medicina. Entre os profissionais, 69 trabalhavam na assistência aos pacientes, e esta foi a composição: 25 enfermeiros, 23 técnicos de enfermagem, 15 médicos, 6 fisioterapeutas e 2 gestores. Os dados demográficos coletados nos questionários estão apresentados na Tabela 1 e podem ser resumidos da seguinte forma: 90,4\% estavam diretamente trabalhando na área específica para os pacientes infectados por COVID-19; o maior percentual de respondentes tinha idade entre 35 e 44 anos (53,4\%); a prevalência maior foi de mulheres, com 83,5\% das respostas. Houve também um predomínio de pessoas brancas (65,7\%); apenas 19,18\% do total morava sozinhos; a maioria tinha pelo menos um filho $(64,3 \%)$; o estado civil mais relatado foi o de união estável/casado (50,6\%); 61,6\% eram moradores da cidade e não precisavam viajar para o trabalho. Todos os participantes tinham concluído pelo menos o ensino técnico e, entre os graduados, a maioria possuía, ao menos, uma pós-graduação $(28,7 \%)$. Trabalhar em mais de um local foi um fator relatado por $72,6 \%$ e, quanto ao número de horas trabalhadas por semana, a resposta de $21 \mathrm{~h}$ a $40 \mathrm{~h}$ mostrou um percentual de 65,75\% (Tabela 1).

RC: 95817

Disponível em: https://www.nucleodoconhecimento.com.br/psicologia/hospitaluniversitario 
Tabela 1: Respostas do questionário sociodemográfico - São Carlos, Brasil, 2020.

\begin{tabular}{|c|c|c|c|}
\hline Variáveis & Categorias & $\begin{array}{l}\text { Frequência } \\
\text { Absoluta }\end{array}$ & $\begin{array}{l}\text { Frequência } \\
\text { Relativa }\end{array}$ \\
\hline Sexo & $\begin{array}{l}\text { Masculino } \\
\text { Feminino }\end{array}$ & $\begin{array}{l}12 \\
61\end{array}$ & $\begin{array}{l}16,44 \% \\
83,56 \%\end{array}$ \\
\hline Gênero & $\begin{array}{l}\text { Masculino } \\
\text { Feminino }\end{array}$ & $\begin{array}{l}12 \\
61\end{array}$ & $\begin{array}{l}16,44 \% \\
83,56 \%\end{array}$ \\
\hline Autodeclaração de etnia/cor & $\begin{array}{l}\text { Branca } \\
\text { Parda } \\
\text { Preta } \\
\text { Amarela } \\
\text { Indígena }\end{array}$ & $\begin{array}{l}48 \\
13 \\
5 \\
2 \\
0\end{array}$ & $\begin{array}{l}65,75 \% \\
17,81 \% \\
6,85 \% \\
2,74 \% \\
0,00 \%\end{array}$ \\
\hline Idade & $\begin{array}{l}18-24 \\
25-34 \\
35-44 \\
45-54 \\
55-64 \\
65 \text { ou + }\end{array}$ & $\begin{array}{l}0 \\
18 \\
39 \\
12 \\
4 \\
0\end{array}$ & $\begin{array}{l}0,00 \% \\
24,66 \% \\
53,42 \% \\
16,44 \% \\
5,48 \% \\
0,00 \%\end{array}$ \\
\hline Estado civil & $\begin{array}{l}\text { Solteiro(a) } \\
\text { Casado(a) } \\
\text { Separado(a) } \\
\text { Divorciado(a) } \\
\text { Viúvo(a) }\end{array}$ & $\begin{array}{l}23 \\
37 \\
3 \\
9 \\
1\end{array}$ & $\begin{array}{l}31,51 \% \\
50,68 \% \\
4,11 \% \\
12,33 \% \\
1,37 \%\end{array}$ \\
\hline Possui filhos? & $\begin{array}{l}\text { Sim } \\
\text { Não }\end{array}$ & $\begin{array}{l}47 \\
26\end{array}$ & $\begin{array}{l}64,38 \% \\
35,62 \%\end{array}$ \\
\hline
\end{tabular}

RC: 95817

Disponível em: https://www.nucleodoconhecimento.com.br/psicologia/hospitaluniversitario 
Local de residência

Número de moradores em seu domicílio (incluindo você)

São Carlos
Ribeirão Preto
Araraquara
Ibaté
São Paulo
Botucatu
Dourado
Descalvado
Brotas
Rincão
Analândia
Lençóis paulista
Pirassununga
Valinhos

\section{1}

2

3

4

5

7
45

2

10

2

3

2

2

1

1

1

1

1

1

1

14

14

15

16

1

1
$61,64 \%$

$2,74 \%$

$13,70 \%$

$2,74 \%$

$4,11 \%$

$2,74 \%$

$2,74 \%$

$1,37 \%$

$1,37 \%$

$1,37 \%$

$1,37 \%$

$1,37 \%$

$1,37 \%$

$1,37 \%$

$19,18 \%$

$19,18 \%$

$20,55 \%$

$21,92 \%$

$1,37 \%$

$1,37 \%$

RC: 95817

Disponível em: https://www.nucleodoconhecimento.com.br/psicologia/hospitaluniversitario 


\begin{tabular}{|c|c|c|c|}
\hline \multirow[t]{8}{*}{ Escolaridade } & $\begin{array}{l}\text { Ensino Médio } \\
\text { incompleto }\end{array}$ & 0 & $0,00 \%$ \\
\hline & $\begin{array}{l}\text { Ensino Médio completo } \\
\text { Ensino Superior }\end{array}$ & 9 & $12,33 \%$ \\
\hline & $\begin{array}{l}\text { incompleto } \\
\text { Ensino Superior }\end{array}$ & 7 & $9,59 \%$ \\
\hline & completo & 14 & $19,18 \%$ \\
\hline & Pós-graduação & 21 & $28,77 \%$ \\
\hline & Mestrado & 13 & $17,81 \%$ \\
\hline & Doutorado & 7 & $9,59 \%$ \\
\hline & Pós-doutorado & 2 & $2,74 \%$ \\
\hline \multirow[t]{9}{*}{ Profissão exercida no HU } & Técnico de enfermagem & 23 & $31,51 \%$ \\
\hline & Enfermeiro & 25 & $34,25 \%$ \\
\hline & Médico & 15 & $20,55 \%$ \\
\hline & Interno de medicina & 2 & $2,74 \%$ \\
\hline & Fisioterapeuta & 6 & $8,22 \%$ \\
\hline & Nutricionista & 0 & $0,00 \%$ \\
\hline & Fonoaudiólogo & 0 & $0,00 \%$ \\
\hline & Chefia do setor & 1 & $1,37 \%$ \\
\hline & enfermagem & 1 & $1,37 \%$ \\
\hline \multirow{2}{*}{$\begin{array}{l}\text { Está trabalhando } \\
\text { diretamente no combate ao } \\
\text { COVID-19? }\end{array}$} & Sim & 66 & $90,41 \%$ \\
\hline & Não & 7 & $9,59 \%$ \\
\hline \multirow{2}{*}{$\begin{array}{l}\text { Trabalha para mais de uma } \\
\text { instituição? }\end{array}$} & Sim & 20 & $27,40 \%$ \\
\hline & Não & 53 & $72,60 \%$ \\
\hline \multirow{5}{*}{$\begin{array}{l}\text { Número de horas } \\
\text { trabalhadas por semana }\end{array}$} & 20 ou menos & 1 & $1,37 \%$ \\
\hline & De 21 a 40 & 48 & $65,75 \%$ \\
\hline & De 41 a 50 & 8 & $10,96 \%$ \\
\hline & De 50 a 60 & 11 & $15,07 \%$ \\
\hline & Mais de 60 & 5 & $6,85 \%$ \\
\hline
\end{tabular}

Fonte: autores.

A distribuição das dimensões e seus respectivos percentuais estão descritos na Tabela 2. Uma característica comum entre os técnicos de enfermagem (TE), os enfermeiros $(E)$ e os médicos $(M)$ foi a alta realização profissional nas três profissões (69\%, 76\% e 60\%, respectivamente), sendo que a despersonalização também foi alta $(65 \%, 48 \%$ e $73 \%$, respectivamente). No caso de fisioterapeutas $(F)$, as três dimensões apareceram na metade dos respondentes. Para dois gestores (GE) e

RC: 95817

Disponível em: https://www.nucleodoconhecimento.com.br/psicologia/hospitaluniversitario 
dois alunos (IM) participantes, a despersonalização foi a dimensão que esteve presente.Fonte: autores.

Tabela 2: Dimensões da SB e seus percentuais - São Carlos, Brasil, 2020.

\begin{tabular}{|c|c|c|c|c|c|c|c|}
\hline \multirow{2}{*}{\multicolumn{2}{|c|}{ Dimensões }} & \multicolumn{6}{|c|}{ Profissões } \\
\hline & & \multirow{2}{*}{$\begin{array}{l}\text { TE \% } \\
56,52\end{array}$} & \multirow{2}{*}{$\begin{array}{l}E \% \\
32\end{array}$} & \multirow{2}{*}{$\begin{array}{l}\text { M \% } \\
46,67\end{array}$} & \multirow{2}{*}{$\begin{array}{l}\text { IM \% } \\
50\end{array}$} & \multirow{2}{*}{$\begin{array}{l}\mathrm{F} \% \\
50\end{array}$} & \multirow{2}{*}{$\begin{array}{c}\text { GE \% } \\
0,00\end{array}$} \\
\hline EE & Positivo & & & & & & \\
\hline & Negativo & 43,48 & 68 & 53,33 & 50 & 50 & 100 \\
\hline \multirow[t]{2}{*}{ DE } & Positivo & 65,22 & 48 & 73,33 & 100 & 50 & 100 \\
\hline & Negativo & 34,78 & 52 & 26,67 & 0 & 50 & 0,00 \\
\hline \multirow[t]{2}{*}{$\mathrm{RP}$} & Positivo & 30,44 & 24 & 40 & & 50 & 100 \\
\hline & Negativo & 69,56 & 76 & 60 & 0,00 & 50 & 0,00 \\
\hline
\end{tabular}

Fonte: autores.

A associação entre as dimensões da SB é apresentada na Tabela 3. De acordo com os critérios do MBI-HSS, em cinco técnicos de enfermagem, três enfermeiros, uma médica e uma fisioterapeuta, foram encontradas as três dimensões positivas nas respostas, o que representa a presença da SB. Fonte: autores.

RC: 95817

Disponível em: https://www.nucleodoconhecimento.com.br/psicologia/hospitaluniversitario 
Tabela 3 - Distribuição das três dimensões do MBI-HSS e os respectivos percentuais para cada categoria profissional - São Carlos, Brasil, 2020.

\begin{tabular}{|c|c|c|c|c|c|c|}
\hline $\begin{array}{l}\text { Categoria } \\
\text { Profissional }\end{array}$ & $\mathrm{N}^{\circ}$ & $\begin{array}{l}\text { Uma } \\
\text { dimensão } \\
\text { positiva (\%) }\end{array}$ & $\mathrm{N}^{0}$ & $\begin{array}{l}\text { Duas } \\
\text { dimensões } \\
\text { positivas (\%) }\end{array}$ & $\mathrm{N}^{\circ}$ & $\begin{array}{l}\text { Três } \\
\text { dimensões } \\
\text { positivas } \\
\text { (Síndrome de } \\
\text { Burnout) }(\%)\end{array}$ \\
\hline Enfermeiros & 7 & 28 & 6 & 24 & 3 & 12 \\
\hline Médicos & 6 & 40 & 8 & 53,3 & 1 & 6 \\
\hline $\begin{array}{l}\text { Técnicos de } \\
\text { Enfermagem }\end{array}$ & 7 & 30 & 7 & 30 & 5 & 21,7 \\
\hline Fisioterapeutas & 2 & 33,3 & 1 & 16,6 & 1 & 16,6 \\
\hline Gestores & 1 & 50 & 0 & 0 & 0 & 0 \\
\hline $\begin{array}{l}\text { Internos de } \\
\text { Medicina }\end{array}$ & 1 & 50 & 1 & 50 & 0 & 0 \\
\hline $\begin{array}{l}\text { Total na } \\
\text { amostra }\end{array}$ & 24 & & 23 & & 10 & \\
\hline
\end{tabular}

Fonte: autores.

A Tabela 4 mostra a correlação entre variáveis sociodemográficas e dimensões MBIHSS para cada categoria profissional. No caso dos técnicos de enfermagem, foi observada uma associação positiva entre os casados/união estável e realização profissional $(\mathrm{OR}=2,25 ; \mathrm{IC} 95 \%[0,2 ; 17,7] ; p=0,04)$. Para os enfermeiros, houve uma associação positiva entre ter filho e a despersonalização ( $O R=2,48$; IC $95 \%$ $[1,11 ; 5,57] ; p=0,01)$. Quanto aos médicos, cada dimensão teve uma associação positiva: o fato de ter mais de um emprego impactou o esgotamento emocional (OR $=0,5$; IC 95\% $[0,04 ;, 7,7] ; p=0,02)$; o fato de ser casado contribuiu para uma maior despersonalização ( $O R=2,5 ;$ IC 95\% $[0,19 ; 32,1] ; p=0,01)$. $E$, no sexo feminino, apareceu uma maior realização profissional $(\mathrm{OR}=2,5$; IC 95\% $[0,19 ; 32,1] ; \mathrm{p}=$ 0,01). A validação estatística de fisioterapeutas, gestores e alunos não foi significativa. Fonte: autores.

RC: 95817

Disponível em: https://www.nucleodoconhecimento.com.br/psicologia/hospitaluniversitario 
Tabela 4 - Correlação entre variáveis sociodemográficas e dimensões MBI-HSS para cada categoria profissional. - São Carlos, Brasil, 2020.

\begin{tabular}{|c|c|c|c|c|c|c|c|c|c|c|}
\hline \multirow[t]{2}{*}{ Variável } & \multirow[t]{2}{*}{ Categoria } & \multicolumn{3}{|c|}{$\mathrm{EE}$} & \multicolumn{3}{|c|}{$\mathrm{DE}$} & \multicolumn{3}{|c|}{$\mathrm{RP}$} \\
\hline & & $\mathrm{OR}$ & (IC 95\%) & p-valor & $\overline{O R}$ & (IC 95\%) & p-valor & $\overline{\mathrm{OR}}$ & (IC 95\%) & p-valor \\
\hline $\begin{array}{l}\text { Téc. } \\
\text { enfermag } \\
\text { em }\end{array}$ & & & & & & & & & & \\
\hline $\begin{array}{l}\text { Estado } \\
\text { Civil }\end{array}$ & Solteiro & 1000 & & & & 1000 & & 1.000 & & \\
\hline & Casado & 1,042 & $\begin{array}{l}(0,18 ; \\
6,12)\end{array}$ & 0,964 & 1,250 & $\begin{array}{l}(0,067 \\
12,25)\end{array}$ & 0,881 & 2,250 & $\begin{array}{l}(0,285 ; \\
17,759)\end{array}$ & 0,042 \\
\hline
\end{tabular}

\begin{tabular}{|c|c|c|c|c|c|c|c|c|c|c|}
\hline \multirow[t]{2}{*}{ Variável } & \multirow[t]{2}{*}{ Categoria } & \multicolumn{2}{|r|}{$\mathrm{EE}$} & \multirow[b]{2}{*}{ p-valor } & \multicolumn{3}{|c|}{$\mathrm{DE}$} & \multicolumn{3}{|c|}{$\mathrm{RP}$} \\
\hline & & $\overline{\mathrm{OR}}$ & (IC 95\%) & & OR & (IC 95\%) & p-valor & $\overline{\mathrm{OR}}$ & (IC 95\%) & p-valor \\
\hline \multirow[t]{2}{*}{$\begin{array}{l}\text { Enfermei } \\
\text { o } \\
\text { Estado } \\
\text { Civil }\end{array}$} & Solteir & 1000 & & & 1000 & & & 1000 & & \\
\hline & & 1,500 & $\begin{array}{l}(0,44 ; \\
5,15)\end{array}$ & 0,646 & 1,125 & $\begin{array}{l}(0,372 ; \\
3,406)\end{array}$ & 1,000 & 0,296 & $\begin{array}{l}(0,047 ; \\
1,867)\end{array}$ & 0,016 \\
\hline \multirow[t]{2}{*}{$\begin{array}{l}\text { Possui } \\
\text { filhos? }\end{array}$} & Não & 1,000 & & & 1000 & & & 1000 & & \\
\hline & Sim & 0,346 & $(0,08 ; 1,5)$ & 0,178 & 2,489 & $\begin{array}{l}(1,111 ; \\
5,574)\end{array}$ & 0,015 & 0,890 & $\begin{array}{l}(0,538 ; \\
1,472)\end{array}$ & 1,000 \\
\hline
\end{tabular}

\begin{tabular}{|c|c|c|c|c|c|c|c|c|c|c|}
\hline \multirow[t]{2}{*}{ Variável } & \multirow[t]{2}{*}{ Categoria } & \multicolumn{3}{|c|}{$\mathrm{EE}$} & \multicolumn{3}{|c|}{ DE } & \multicolumn{3}{|c|}{$\mathrm{RP}$} \\
\hline & & $\overline{O R}$ & (IC 95\%) & p-valor & OR & (IC 95\%) & p-valor & $\overline{O R}$ & (IC 95\%) & p-valor \\
\hline \multicolumn{11}{|l|}{ Médico } \\
\hline \multirow{3}{*}{$\begin{array}{l}\text { Estado } \\
\text { Civil }\end{array}$} & & & & & & & & & & \\
\hline & Solteiro & 1,000 & & & 1,000 & & & 1,000 & & \\
\hline & Casado & 6,250 & $77,49)$ & 0,287 & 2,500 & $32,19)$ & 0,011 & - & - & 1,000 \\
\hline \multirow[t]{2}{*}{ Sexo } & Masculino & 1,000 & & & 1,000 & & & 1,000 & & \\
\hline & Feminino & 1,429 & $\begin{array}{l}(0,10 \\
20,44)\end{array}$ & & - & - & - & 0,500 & $\begin{array}{l}(0,28 \\
0,88)\end{array}$ & 0,025 \\
\hline Trabalha & Não & 1,000 & & & 1,000 & & & 1,000 & & \\
\hline instituição & Sim & 0.571 & $\begin{array}{l}(0,04 ; \\
774)\end{array}$ & 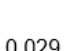 & 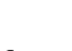 & 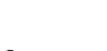 & 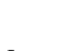 & 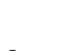 & 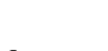 & 0 \\
\hline
\end{tabular}

Fonte: autores.

RC: 95817

Disponível em: https://www.nucleodoconhecimento.com.br/psicologia/hospitaluniversitario 


\section{DISCUSSÃO}

Conforme os critérios adotados para a análise das três dimensões do MBI-HSS e considerando apenas os profissionais atuando no combate à COVID-19 das categorias estatisticamente relevantes para o estudo (enfermeiros, técnicos de enfermagem e médicos), as respostas obtidas neste levantamento mostraram uma moderada prevalência de exaustão emocional (média de 48,27\%, sendo a maior em técnicos de enfermagem, com 56,52\%) e uma alta prevalência de despersonalização (média de 65,51\%, sendo a maior em médicos, com 73,3\%). Contudo, como os resultados para baixa realização pessoal ficou, em média, em $32,75 \%$, a prevalência da SB entre os profissionais de saúde do hospital estudado foi de cerca de $14 \%$. A literatura mundial apresenta níveis mais elevados de SB em unidades de saúde (33,8\%) mesmo sem a presença da COVID-19 (CAÑADES et al., 2015; SUÑERSOLER et al., 2014). Estudos chineses iniciais sobre saúde mental de profissionais de saúde assistenciais da linha de frente do enfrentamento à COVID-19 mostraram que a prevalência aumenta conforme cresce a demanda de pacientes aos serviços de saúde (LAl et al., 2020; CHEN et al., 2020). Depois, com o avanço da pandemia, estudos em outros países também apontaram uma maior frequência de sinais de exaustão e SB em profissionais da linha de frente (SASAGOHAR et al., 2020; MATSUO et al., 2020; BARELLO et al., 2020; KOH, 2020; HORTA et al., 2021; FREITAS et al., 2021).

Neste estudo, ocorreram algumas limitações. O poder estatístico da análise foi limitado pelo tamanho reduzido da amostra. A amostra, entretanto, corresponde a $25 \%$ dos profissionais de saúde do hospital. A grande maioria dos respondentes realizava o trabalho na área reservada para 0 atendimento de pacientes com COVID-19. Como não há estudo anterior na instituição sobre a prevalência de SB, não é possível fazer uma análise comparativa da prevalência antes da COVID-19. Assim, a amostra é apenas representativa da presença de SB nos profissionais durante o período da pandemia. Outra limitação é o caráter local, que foi um dos focos do estudo, mas que não pode ser generalizado para outras regiões do país.

RC: 95817

Disponível em: https://www.nucleodoconhecimento.com.br/psicologia/hospitaluniversitario 
Apesar de o estudo ter se estendido a outros profissionais, como fisioterapeutas, gestores e até estudantes de medicina, houve poucos participantes, assim os resultados para essas categorias não tiveram significância estatística. Em relação a técnicos de enfermagem, enfermeiros e médicos, há a possibilidade de o resultado estar influenciado pelo ambiente de exposição à pandemia, o que pode resultar em uma supervalorização de sentimentos. Como o estudo foi realizado por meio de um questionário, a iniciativa de responder ao mesmo pode ter sido influenciada pela afinidade que os participantes têm com a profissão. Os resultados obtidos mostram, entretanto, uma paridade com outros estudos brasileiros (HORTA et al., 2021; FREITAS et al., 2021).

Nos resultados de cada categoria profissional, foi verificado que a presença de SB em técnicos ficou em 21,7\%. Um pouco menor que a encontrada por Freitas et al. em 2020 (FREITAS et al., 2021): em um estudo com técnicos de enfermagem de terapia intensiva que estão atuando na linha de frente da pandemia de COVID-19, a prevalência da SB foi de 25,5\%. Fatores sociodemográficos, ocupacionais e comportamentais mostraram-se como preditores da síndrome. Pelos resultados deste trabalho, não foi possível fazer esse tipo de associação, embora os níveis de despersonalização fossem altos. Além disso, os técnicos de enfermagem foram os colaboradores que apresentaram o maior percentual de exaustão emocional (EE) e de baixa realização profissional (RP). A rotina diária envolvida no trabalho dos técnicos de enfermagem é desgastante, leva à fadiga e pode ser a responsável pelo alto índice de despersonalização (VASCONCELOS, 2016).

No Brasil, estudos da SB em enfermeiros são mais frequentes do que em outras categorias de profissionais de saúde. Para enfermeiros, estudos sobre SB mostram uma prevalência em torno de 25-50\% (MATSUO et al., 2020; AZEVEDO et al., 2019). Apesar de os resultados mostrarem moderadas taxas de exaustão emocional e despersonalização, a prevalência de SB entre enfermeiros foi de $12 \%$ no hospital analisado. Um dado que foi observado e não está presente em literatura foi a associação positiva entre a despersonalização e os profissionais com filhos. Aqui pode ser considerado um fator de sobrecarga.

RC: 95817

Disponível em: https://www.nucleodoconhecimento.com.br/psicologia/hospitaluniversitario 
Em relação aos médicos, a incidência do SB foi de $6 \%$, a qual é baixa em comparação à literatura (CHEN et al., 2020; WU et al., 2020). Apesar disso, foram verificadas uma alta despersonalização e uma alta realização profissional. $O$ fato de o profissional ter mais de um emprego teve impacto na exaustão emocional $(\mathrm{OR}=$ 0,5; IC 95\% [0,04; ,7,7]; $p=0,02)$. Índices mais altos de exaustão emocional são encontrados com uma maior carga de trabalho. $O$ fato de o profissional ser casado contribuiu para uma maior despersonalização $(\mathrm{OR}=2,5$; IC 95\% [0,19; 32,1]; $\mathrm{p}=$ $0,01)$. Estudos da SB com médicos são muito heterogêneos, e várias causas são fontes de desvio, como número de empregos, especialidade e carga horária de trabalho. Índices mais altos de exaustão emocional são encontrados com uma maior carga de trabalho (FREITAS et al., 2021). A despersonalização pode estar vinculada a um menor vínculo do profissional com a instituição, já que o perfil dos médicos da instituição é de não trabalhar diariamente no mesmo local.

Em relação específica ao atendimento a pacientes com COVID-19, há um estudo recente de Dinibutun (DINIBUTUN, 2020) em que médicos apresentaram baixa prevalência de SB. O resultado sugeria que médicos ativamente envolvidos no enfrentamento da COVID-19 apresentavam um forte sentimento de realização pessoal e satisfação com o trabalho, pois percebiam os resultados imediatos do cuidado com as pessoas infectadas e, consequentemente, sofriam menos de SB. Além disso, o estudo recente de Yıldırım e Solmaz demonstrou a importância do papel da resiliência na mediação do desenvolvimento da SB relacionada à COVID19, sendo que a resiliência atua de maneira contrária ao estresse no desenvolvimento da síndrome no contexto de pandemia (SOLMAZ et al., 2020)

Outro aspecto que possivelmente impacta o resultado do estudo é o fato de o hospital analisado receber pacientes cuja procedência é necessariamente de outro serviço de saúde da cidade ("porta fechada"), não estando sujeito à demanda espontânea da população. Dessa forma, o hospital está menos sujeito a flutuações bruscas de procura por atendimento e superlotação, o que pode resultar em profissionais menos sobrecarregados em função do trabalho. 
A SB é um processo que decorre da interação entre o ambiente de trabalho e as características pessoais. A definição mais utilizada foi proposta por Maslach e Jackson em 1981 (MASLACH et al., 2001). A exaustão emocional é considerada um fator central da SB. Caracteriza-se pelo emocional e pelo senso de falta de energia, mostrando associação inversa com o desempenho do trabalho. A EE está frequentemente relacionada a demandas excessivas e conflitos pessoais, predominantemente em pessoas com grau superior (TAMAYO et al., 2002). A despersonalização refere-se à perda de motivação, à ansiedade, à irritabilidade e ao idealismo reduzido. A área da saúde é considerada uma atividade que exige estabilidade emocional e capacidade de expressar emoções (EMBRIACO et al., 2007). A baixa realização profissional está relacionada a um sentimento de incompetência e inadequação, à autoavaliação negativa e à queda de produtividade. Outro fator que pode influenciar o sentimento de redução da realização profissional é a falta de reconhecimento no trabalho (MASLACH et al., 2001; ALMEIDA et al., 2009). A SB é um processo que decorre da interação entre o ambiente de trabalho e a realização pessoal. As descobertas deste estudo podem ser explicadas pelo perfil da instituição investigada. As atividades assistenciais de saúde podem gerar altas demandas emocionais no profissional. Sobrecarga de trabalho em técnicos, enfermeiros e médicos aumenta o turnover de profissionais, o que impacta a qualidade do atendimento assistencial (SILVA et al., 2008). Apesar de o propósito de ajudar outras pessoas ser reconhecido como um objetivo nobre, a relação entre um profissional e os pacientes e a fina distinção entre envolvimento profissional e pessoal são permeadas pela ambiguidade (ALMEIDA et al., 2009).

A pandemia de COVID-19 colocou desafios aos serviços de saúde e forçou mudanças de rotinas e da atuação de profissionais. As adequações de rotinas e de espaços físicos foram necessárias, além de ter sido preciso também oferecer apoio emocional às equipes. O MBI-HSS não tem poder diagnóstico para SB, que deve ser diagnosticada por psiquiatra experiente (VASCONCELOS, 2016). Contudo a ferramenta, além de ser um complemento ao diagnóstico clínico, mostra o impacto 
que o ambiente tem no desgaste emocional. Assim, torna-se um método de avaliação importante para os serviços durante o período da pandemia.

\section{CONCLUSÃO}

A pesquisa teve como objetivo avaliar a prevalência da SB apresentada pelos profissionais de saúde no período de pandemia de COVID-19 e se alguns fatores sociodemográficos poderiam ter uma contribuição para o desenvolvimento da síndrome. Nas três categorias profissionais em que foi possível fazer o levantamento, foram encontrados oito trabalhadores com as três dimensões da SB (prevalência de 14\% na amostra). Apesar da exaustão emocional e da despersonalização estarem altas, havia também alta realização pessoal, independentemente da categoria. É possível que a afinidade dos profissionais com o serviço, bem como o sentimento de realização pessoal pela atuação na pandemia, tenha contribuído para o resultado. É possível também que estratégias de gestão possam diminuir os níveis de exaustão emocional e despersonalização, tais como mitigação de cargas de trabalho prolongadas e condições adequadas de repouso (CHUNG et al., 2020; CHEN et al., 2020). Ademais, características próprias do funcionamento da instituição podem ter um papel significante no desenvolvimento da SB entre os profissionais neste estudo.

\section{REFERÊNCIAS}

AZEVEDO, Kely Cristina Carneiro de et al. National scientific production on Burnout Syndrome in ICU nurses and physicians: a bibliometric study. Revista da Associação Médica Brasileira, v. 65, n. 5, p. 722-729, 2019.

BARELLO, Serena; PALAMENGHI, Lorenzo; GRAFFIGNA, Guendalina. Burnout and somatic symptoms among frontline healthcare professionals at the peak of the Italian COVID-19 pandemic. Psychiatry research, v. 290, p. 113129, 2020.

BRIDGERMAN, Patrick; BRIDGERMAN, Mary; BARONE, Joseph. Burnout syndrome 
among healthcare professionals. Am J Health-Syst Pharm.; 75:147-52. 2018.

CAÑADAS-DE LA FUENTE, Guillermo A. et al. Risk factors and prevalence of burnout syndrome in the nursing profession. International journal of nursing studies, v. 52, n. 1, p. 240-249, 2015.

CHEN, Qiongni et al. Mental health care for medical staff in China during the COVID19 outbreak. The Lancet Psychiatry, v. 7, n. 4, p. e15-e16, 2020.

CHUNG, Joseph PY et al. Staff mental health self-assessment during the COVID-19 outbreak. East Asian Archives of Psychiatry, v. 30, n. 1, p. 34, 2020.

CODO, Wanderley. Um Diagnóstico do Trabalho - Em Busca de Prazer. Por uma Psicologia do Trabalho: Ensaios Recolhidos. São Paulo: Casa do Psicólogo, p. 75-97. 2006.

DA SILVA, Jorge Luiz Lima et al. Psychosocial factors and prevalence of burnout syndrome among nursing workers in intensive care units. Revista Brasileira de terapia intensiva, v. 27, n. 2, p. 125, 2015.

DE ALMEIDA, Karine Moreira; CARLOTTO, Mary Sandra; DE SOUZA, Lúcia Azambuja. Síndrome de Burnout em funcionários de uma fundação de proteção e assistência social. Revista Psicologia: Organizações e Trabalho, v. 9, n. 2, p. 8696, 2009.

DE OLIVEIRA VASCONCELOS FILHO, Paulo. Burnout Syndrome Among Health Workers At A Brazilian Public Hospital Run By A Social Health Organization. World Journal of Research and Review (WJRR), v. 3, p. 32-37. 2016.

DINIBUTUN, Sait Revda. Factors associated with burnout among physicians: An evaluation during a period of COVID-19 pandemic. Journal of Healthcare Leadership, v. 12, p. 85, 2020. 
EBLING, Márcia; CARLOTTO, Mary Sandra. Burnout syndrome and associated factors among health professionals of a public hospital. Trends in psychiatry and psychotherapy, v. 34, n. 2, p. 93-100, 2012.

EMBRIACO, Nathalie; PAPAZIAN, Laurent; KENTISH-BARNES, Nancy; POCHARD, Elie. Burnout syndrome among critical care healthcare workers. Curr Opin Crit Care 13:482-488. 2007.

FREITAS, Ronilson Ferreira et al. Preditores da síndrome de Burnout em técnicos de enfermagem de unidade de terapia intensiva durante a pandemia da COVID-19. Jornal Brasileiro de Psiquiatria, v. 70, n. 1, p. 12-20, 2021

GREENGLASS, Esther R.; BURKE, Ronald J.; FIKSENBAUM, Lisa. Workload and burnout in nurses. Journal of community \& applied social psychology, v. 11, n. 3 , p. 211-215, 2001.

HORTA, Rogério Lessa et al. O estresse e a saúde mental de profissionais da linha de frente da COVID-19 em hospital geral. Jornal Brasileiro de Psiquiatria, v. 70, n. 1, p. 30-38, 2021.

LAI, Jianbo et al. Factors associated with mental health outcomes among health care workers exposed to coronavirus disease 2019. JAMA network open, v. 3, n. 3, p. e203976-e203976, 2020.

LAUTERT, L. Professional fatigue: empirical study with hospital nurses. Revista gaucha de enfermagem, v. 18, n. 2, p. 133-144, 1997.

LIU, Zhonghua. The epidemiological characteristics of an outbreak of 2019 novel coronavirus diseases (COVID-19) in China. Epidemiology Working Group for NCIP Epidemic Response, Chinese Center for Disease Control and Prevention; 41(2):145-51. 2020. 
MASLACH, Christina; JACKSON, Susan. Burnout in organizational settings. Applied Soc Psychol Ann; 5:133-153. 1984.

MASLACH, Christina; JACKSON, Susan. The measurement of experienced Burnout. J Occup Behav; 2: 99-1. 1981.

MASLACH, Christina; SCHAUFELI, Wilmar; LEITER, Michael. Job burnout. Ann Rev Psychol; 52(1): 397-422. 2001.

MATSUO, Takahiro et al. Prevalence of health care worker burnout during the coronavirus disease 2019 (COVID-19) pandemic in Japan. JAMA network open, v. 3, n. 8, p. e2017271-e2017271, 2020.

ROTENSTEIN, Lisa S. et al. Prevalence of burnout among physicians: a systematic review. Jama, v. 320, n. 11, p. 1131-1150, 2018.

SASANGOHAR, Farzan et al. Provider burnout and fatigue during the COVID-19 pandemic: lessons learned from a high-volume intensive care unit. Anesthesia and analgesia, 2020.

SHANAFELT, Tait; RIPP, Jonathan; TROCKEL, Mickey. Understanding and addressing sources of anxiety among health care professionals during the COVID-19 pandemic. Jama, v. 323, n. 21, p. 2133-2134, 2020.

SOCIETY OF CRITICAL CARE MEDICINE et al. Clinicians report high stress in COVID-19 response. 2020.

SUÑER-SOLER, Rosa et al. The consequences of burnout syndrome among healthcare professionals in Spain and Spanish speaking Latin American countries. Burnout research, v. 1, n. 2, p. 82-89, 2014.

SOLMAZ, Fatma; YILDIRIM, Murat. COVID-19 burnout, COVID-19 stress and resilience: Initial psychometric properties of COVID-19 Burnout Scale. Death Studies, p. 1-9, 2020.

RC: 95817

Disponível em: https://www.nucleodoconhecimento.com.br/psicologia/hospitaluniversitario 
TAMAYO, Maurício. Relationship between burnout syndrome and the organizational values in nursing staff of two public hospitals. [Master degree]. Brasilia: Brasilia University; 1997.

TAMAYO, Maurício; TROCCOLI, Bartholomeu. Exaustão emocional: relações com a percepção de suporte organizacional e com as estratégias de coping no trabalho. Estudos de Psicologia, 7(1), 37-46. 2002.

TRINDADE, Letícia de Lima et al. Estresse e síndrome de burnout entre trabalhadores da equipe de Saúde da Família. Acta Paulista de Enfermagem, v. 23, n. 5, p. 684-689, 2010.

WORLD HEALTH ORGANIZATION. Coronavirus disease 2019 pandemic. Disponível em: https://www.who.int/emergencies/diseases/novel-coronavirus-2019 Acesso em: abril 2020.

WU, Yuan et al. A comparison of burnout frequency among oncology physicians and nurses working on the frontline and usual wards during the COVID-19 epidemic in Wuhan, China. Journal of pain and symptom management, v. 60, n. 1, p. e60e65, 2020.

Enviado: Maio, 2021.

Aprovado: Agosto, 2021. 